\title{
Reconstructing Social Interactions Using an unreliable Wireless Sensor Network
}

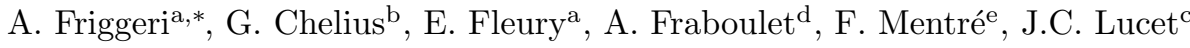 \\ ${ }^{a}$ LIP UMR 5668/ENS de Lyon, DNET/INRIA, Université de Lyon \\ ${ }^{b}$ DNET/INRIA, LIP UMR 5668/ENS de Lyon, Université de Lyon \\ ${ }^{c}$ Hôpital Bichat - Claude Bernard, AP-HP, Université Paris-Diderot - Paris VII, Paris \\ ${ }^{d}$ CITI/INSA de Lyon, AMAZONES/INRIA, Université de Lyon \\ e UMR 738 INSERM, Université Paris Diderot
}

\begin{abstract}
In the very active field of complex networks, research advances have largely been stimulated by the availability of empirical data and the increase in computational power needed for their analysis. These works have led to the identification of similarities in the structures of such networks arising in very different fields, and to the development of a body of knowledge, tools and methods for their study.

While many interesting questions remain open on the subject of static networks, challenging issues arise from the study of dynamic networks. In particular, the measurement, analysis and modeling of social interactions are first class concerns.

In this article, we address the challenges of capturing physical proximity and social interaction by means of a wireless network. In particular, as a concrete case study, we exhibit the deployment of a wireless sensor network applied to the measurement of Health Care Workers' exposure to tuberculosis infected patients in a service unit of the Bichat-Claude Bernard hospital in Paris, France. This network has continuously monitored the presence of all HCWs in all rooms of the service during a 3 month period.

We both describe the measurement system that was deployed and some early analysis on the measured data. We highlight the bias introduced by the measurement system reliability and provide a reconstruction method which not only leads to a significantly more coherent and realistic dataset but also evidences phenomena a priori hidden in the raw data. By this analysis, we suggest that a processing step is required prior to any adequate exploitation of data gathered thanks to a non-fully reliable measurement architecture.
\end{abstract}

Keywords: complex networks; interaction networks; wireless sensor networks; medical applications

\section{Introduction}

Complex networks [12] appear in many contexts: sociology, computer networks, biology, medicine, etc. Their study has shown a rapid growth since the end of the $90 \mathrm{~s}$ when it was revealed $[2,4,8$, $11,13,15]$ that most real-world complex networks

\footnotetext{
${ }^{*}$ Corresponding author: ENS de Lyon, 46 allée d'Italie, 69364 Lyon Cedex 07. Tel: +33472728000

Email addresses: adrien.friggeri@ens-lyon.fr (A Friggeri), guillaume.chelius@inria.fr (G. Chelius), eric.fleury@inria.fr (E. Fleury),

antoine.fraboulet@insa-lyon.fr (A. Fraboulet), france.mentre@inserm.fr (F. Mentré),

jean-christophe.lucet@bch.ap-hop-paris.fr (J.C. Lucet)
}

Preprint submitted to Elsevier offer common non-trivial properties. This observation has generated a substantial amount of attention during the last decade. While a large part of these works have dealt with static networks, a growing area of research is focused on the dynamic aspects of those networks. The introduction of the temporal dimension is motivated by the fact that most real-world complex networks bear an intrinsic evolution whose analysis and modeling is fundamental to the understanding of the underlying phenomenon.

In numerous fields of study, be it epidemiology, sociology or even the study of computer networks such as Delay Tolerant Networks, the central relational concept is the social interaction or the phys-

August 30, 2010 
ical proximity. Getting a grasp of these relationships is a complex task which is generally fulfilled through audits and interviews, two human-centric approaches. As in the first case, an experimenter monitors and reports the observable interactions whereas in the second one data is compiled thanks to the imperfect memory of the subject, it is obvious that both methods not only suffer from a lack of exhaustivity but also deliver data which reliability heavily depends on human factors. These two measurement properties, lack of reliability and lack of exhaustivity, lead to severe limitations in the abovementioned fields of study.

More recently, with the advances in pervasive networks, wireless devices have offered a new and promising opportunity to gather data on social interactions and physical proximity. As a an example, if phone calls are construed as instances of social interactions, phone records owned by telecom companies represent precious sets of data to be analyzed. Surprisingly, not only the communication but the mere ability to communicate can be interpreted in term of physical proximity. In the context or wireless technologies, a communication between two devices is only feasible when both are in range. This relation between radio and physical proximity has been used in several experiments $[1,6,10]$ in order to measure physical interactions and proximity through the exchange of radio packets between laptops, cell phones or dedicated instruments. Thanks to the embedding of communications devices, which allow passive, periodic and automatic measurement, this type of deployment offers the opportunity to attempt nearly exhaustive measurement campaigns.

Although offering exhaustivity, this measurement method does not provide reliability. Indeed, as the radio medium is pervasive, the relation between radio distance - perceived in term of signal attenuation - and physical distance is far from being predictable. It varies in time and space in a pseudorandom way due to physical phenomenon such as fading and shadowing. In consequence, the measurements gathered during such deployments are noisy and must be considered carefully. In particular, they can not be exploited without being first cleaned and the original interaction information being reconstructed. This step is fundamental as analyses and evaluations performed on raw measurements can lead to results and conclusions that vary drastically from the ones obtained given the real interactions.
Unfortunately, analyses and evaluations are too often based on raw measurements without considering the error induced by the measurement system [10] itself. We assess that this methodological shortcut takes root in an over-confidence in computing devices. The assumption that a measurement is not only exhaustive but also reliable due to its nature of computer-gathered data reveals an utter misconception of the measurement apparatus' limits. For the sake of analyses validity, the challenge is thus to process unreliable data using an estimate of the system induced error in order to acquire an accurate picture of the original interactions.

In this paper, we address this issue through a case study, the deployment of a Wireless Sensor Network (WSN) to measure in situ interactions within a medical context. This project was motivated by the study of the Health Care Workers (HCWs) exposure to tuberculosis in their work environment, as described in Section 3. Sections 4 and 5 present the WSN that was set up to record the presence of HCWs within patient rooms in a specific service care unit of the Bichat Hospital (Paris France). An important characteristic of this measurement campaign is its exhaustivity: it was performed in a closed environment, over a closed population and during a long and continuous period of time. That is, the presence of all HCWs of the unit was monitored, every 5 seconds, in all patient rooms of the unit, 24 hours a day, 7 days a week, during a three month period. It represents both a huge and unique data set describing a complex dynamic interaction network. After describing the raw data that were gathered during the deployment in Section 6 , we emphasize on the bias introduced by the measurement system in Section 7 and show that the versatility of the radio medium leads to noisy and unreliable data. We present in Section 8 a method to reconstruct a presence signal using the available data and describe the results in Section 9. We finally conclude and present future works in Section 10 .

\section{Contributions}

In our opinion, this article presents several contributions in the field of physical proximity measurement using wireless devices:

- it describes a WSN deployment for physical proximity measurement performed in a closed 
environment, over a closed population and during a long and continuous period of time;

- it assesses, through an analysis of the raw data, the unreliability of this measurement process;

- it investigates methods to quantify the measurement's error;

- it proposes a method to eliminate false positives and false negatives in the measured data and to recover the original interaction information.

\section{The TubExpo project}

This work was motivated by the AFFSET TubExpo project. This project aims at evaluating the exposure of health care workers to tuberculosis in their work environment.

\subsection{The health care context: Tuberculosis}

Despite the progresses in treatment and prevention, tuberculosis remains a disease in expansion and represents the third cause of death by infectious pathologies in the world. Emerging countries are the most affected, as the VIH epidemic participates to the tuberculosis amplification. In France, the impact of tuberculosis is globally decreasing (9.2 cases for 100000 inhabitants in France) but with large regional disparities. The french situation is paradoxical, with a hygiene level of a developed country but a strong and increasing tuberculosis incidence among the migrating populations. For this reason, the french superior council for public hygiene (CSHPF) has recently recommended the launch of studies about the tuberculosis infection and its transmission factors [7].

In the health care context, if the transmission between patients has been largely documented and is globally controlled, the health care workers (HCWs) exposure remains obscure. HCWs taking care of tuberculosis-infected patients are particularly exposed to the disease, and, if infected, may become important transmission vectors as they expose both colleagues and patients to the risk of infection. In October 2003, six cases of HCW tuberculosis infection were reported in five hospitals of Paris [5].

Data on the tuberculosis transmission has generally been acquired in a social community context. Individual factors associated to the contamination of HCWs in their work environment are not precisely known [14]. Knowledge acquisition on the transmissibility of the tuberculosis bacilli in the hospital environment is limited by two factors: individual contamination evaluation and exposure measurement. The evaluation of these two factors is complicated as they are impacted by several sub-factors. For the exposure measurement, several parameters must be considered: the tuberculosis bacilli concentration in the environing air, the intensity and frequency of contacts between infected patients and HCWs and, finally, the prevention methods used to restrain the transmission.

\subsection{Visit Measurement: methods}

Among these various parameters, the TubExpo project focuses especially on the evaluation of both contacts intensity and frequency between tuberculosis infected patients and HCWs inside the Service of Infectious and Tropical Diseases (SMIT) of the Bichat-Claude Bernard hospital in Paris, France.

In order to measure the exposure of HCWs to the tuberculosis bacilli, the time spent by the HCWs in each patient room of the unit was monitored during a three months period. As individuals infected or suspected to be infected by tuberculosis are subject to strong isolation rules, we made the hypothesis that the exposure area was limited to their rooms. Obviously, a first limit of the measurement validity is the respect of these isolation rules by the infected patients.

Two different methods were envisaged in order to measure the HCWs presence in patient rooms: the first one is based on manual audits whereas the second makes use of a wireless sensor network (WSN).

\subsubsection{Method 1: Audits}

Performing audits is a classical method to gather data on social interactions or work habits, especially in a health care context. Given the human nature of such a measurement, there is no false positive as a visit is never recorded if it did not actually occured. Moreover, this method does not involve any technical aspect and is simple to deploy. However, as a manual recording, this approach is partial because it does not offer a continuous observation. The experimenter has to be present in the unit for the visit to be recorded. As an example, no audit was performed during the night. It is also subject to observations and interpretations: simultaneous visits in different rooms can hardly be handled by a single experimenter. Finally, it is time and human resource consuming. 


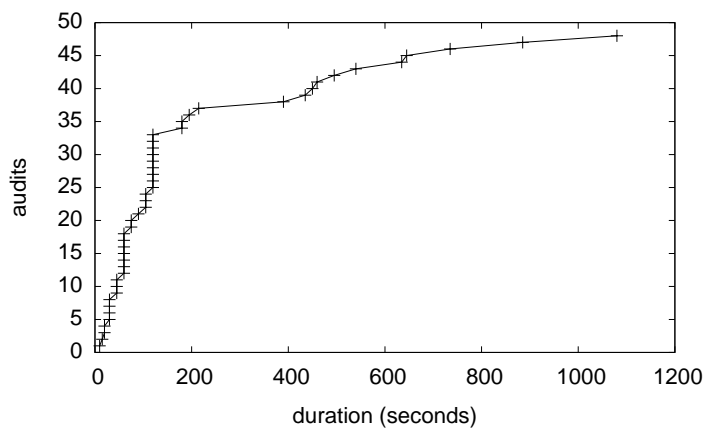

Figure 1: Cumulative distribution of audit durations.

In our case, 48 visits were manually recorded by an experimenter who recorded the relevant room and HCW id, the time of arrival and the duration of each visit. The average duration of a visit was of $3 \mathrm{~min} 26 \mathrm{~s}$, the shortest $10 \mathrm{~s}$ and the longest $18 \mathrm{~min}$. The distribution of durations corresponding to these audits is given in Figure 1.

Obviously, the knowledge of 48 visits over the course of the three months long experiment does not provide any relevant information per se. However, having access to a frame of reference for attested visits can be insightful when correlated with other data, as we will show in Section 7.

\subsubsection{Method 2: WSN}

In order to continuously and exhaustively monitor the presence of HCWs in patient rooms, the second method we used was to deploy a wireless sensor network in the unit of the hospital. This WSN consisted of devices placed in patient rooms and devices handled by the HCWs. Compared to audits, this method offers exhaustivity: it is operating 24 hours a day, 7 days a week, independently of the presence of an experimenter. However, contrarily to what could be primarily thought, its reliability is subject to many factors and the resulting data should not be considered as a perfect image of the interactions reality. As an example, a first error factor, but not the only one, is the human factor and the acceptance of the deployment by the HCWs. Obviously, measurements can not occur if HCWs forget or refuse to handle their sensor device. It is worth noting that within the context of the TubExpo project, only one of the unit's 63 HCWs refused to be enrolled in the experiment and that all rooms were equipped.

\section{A WSN for presence detection}

In this deployment, each room of the SMIT unit in the Bichat-Claude Bernard hospital (Paris, France) was equipped with a sensor node whose task was to continuously listen to the radio medium. More precisely, these fixed sensor nodes were placed at a $2 \mathrm{~m}$ height, under the TV, and plugged to the power line (Figure 2). They were thus provided with an unlimited energy resource.

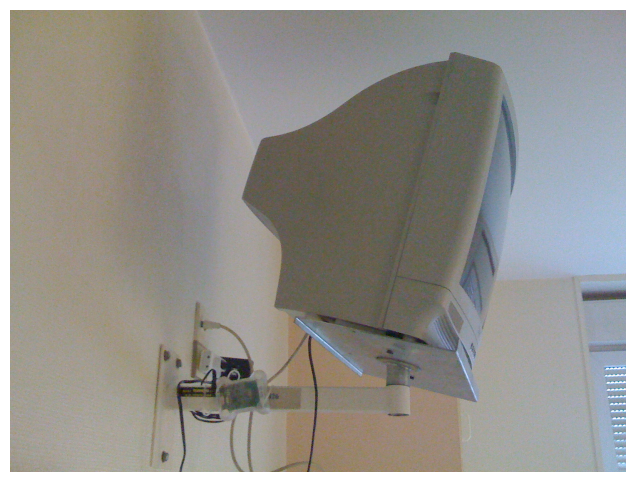

Figure 2: Location of a fixed sensor node in a patient room.

In parallel, each HCW was given an autonomous sensor node they had to carry during their presence in the unit. These mobile sensor nodes were programmed to periodically transmit a radio packet containing their identity. They were under the HCWs responsibility without the possibility to refill their battery during the whole deployment duration. This induced strong energy constraints on their functioning.

Contrarily to previous interactions measurement applications $[1,6,10]$, we chose to deploy an asymmetric network where only the fixed sensor nodes, i.e. the ones installed in the patient rooms, were receiving packets and recording interactions. Briefly, we can advance three main reasons to justify this choice:

- simplicity: in such an asymmetric network, a sensor node does not need to switch between transmission and reception modes, therefore avoiding synchronization issues among others and largely simplifying the sensor node firmwares.

- energy consumption: as the fixed nodes were not energy constrained, they could permanently stay in reception mode and no activity scheduling or duty-cycling policy was required. 
On the contrary, HCW-carried sensor nodes were strongly energy constrained and their radio I/O operations had to be limited to the minimum.

- privacy respect: as we were only interested in the presence of HCWs in patient rooms, we did not need to record the proximity of HCWs between each other. Doing so would not only have required additional privacy respect procedures but also a stronger defense of the project in front of privacy \& work protection committees.

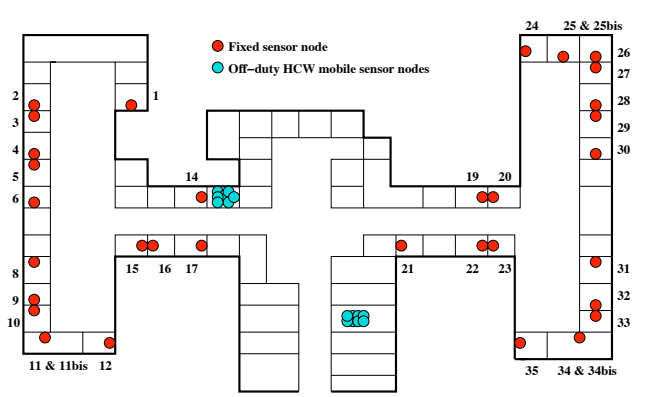

Figure 3: A map of the Service of Infectious and Tropical Diseases (SMIT) at the Bichat-Claude Bernard hospital (Paris, France) together with the fixed sensor node locations (red circles).

The deployed sensor network is depicted in Figure 3. The SMIT is composed of 32 patient rooms which are split in two $\mathrm{T}$ shaped aisles. The SMIT owns $63 \mathrm{HCWs}$, all but one carrying a mobile sensor node. The network was thus composed of 94 sensor nodes that have operated continuously during the three months experiment period. To our knowledge this experiment is the first proximity interaction study performed over a complete closed population during a so long continuous period of time: all but one HCWs of the unit carried a sensor node, each room of the unit was monitored and HCWs were not supposed to leave the unit during their duty.

\section{Hardware and protocol details}

\subsection{Hardware}

The deployment was conducted using WSN430 sensor nodes [9]. Their internal architecture is classical and similar to commercially-available sensor

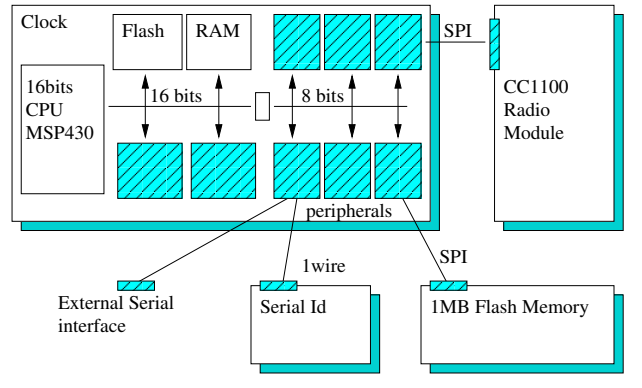

Figure 4: A simplistic schematic of a WSN430 node.

nodes. Very basically, they are composed of a micro-controller, a RF chipset, a flash memory, several crystals, a lithium-ion battery and an on-board PCB antenna (Figure 4). The micro-controller belongs to the TI MSP430 family. The RF chipset is a TI CC1100 operating at the $868 \mathrm{MHz}$ frequency, with a 2 -FSK modulation and a $119 \mathrm{Kbps}$ baud-rate. The flash memory storage size is $1 \mathrm{Mb}$. Figure 5 illustrates a WSN430 node in its plastic packaging.

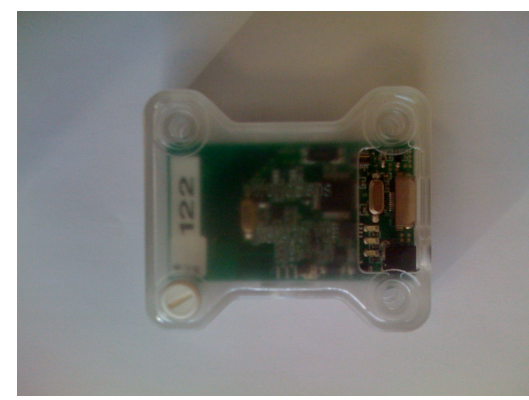

Figure 5: A WSN430 sensor node.

\subsection{Neighbor discovery protocol}

Proximity detection was achieved through the periodical emission of Hello packets. This strategy has the merit of simplicity and has been used in other deployments [3]. It is quite similar to the Bluetooth base-band layer inquiry method used in [10]. However, contrarily to these previous works, we did not implement any random access scheme to the radio medium but rather set up a deterministic TDMA scheme. Under this scheme, the timeline is divided in windows of $W$ seconds and each window is split in $\frac{W}{100}$ time slots. Each mobile sensor node owns a time slot it uses to transmit its Hello packet. Synchronization of the mobile nodes was performed at the experiment startup.

As described in [3], the performance of this very simple neighbor discovery protocol, and thus the 
quality of the proximity detection, largely relies on the dimensioning of the protocol parameters. In our case, the parameters are the window duration $W$, the transmission power $T$ and reception level thresholds $\left\{R_{i}\right\}$. The parameters values were chosen after a pre-deployment dimensioning phase during which different scenarios were explored and several parameter value sets experimented. The final values are given in Section 5.4. We also based our choices on the HCWs recommendations and their work habits observation. For example, it appeared quickly that most of the $\mathrm{HCW}$ presences in patient rooms were brief, around a few minutes, requiring a short $W$ duration to ensure some reliability in the discovery process. As a comparison, the $120 \mathrm{~s}$ period used in [10] is far too long to detect most of the HCWs presences in patient rooms.

\subsection{Versatility of the radio medium}

Three other major observations were made during this pre-deployment dimensioning phase:

- the system suffers an important packet loss, even at short ranges;

- the relation between radio signal attenuation and physical distance is weak;

- the radio signal attenuation is not predictable and can largely vary.

Figure 6 illustrates the two first claims. It depicts the relation between distance and the Received Signal Strength (RSSI) and the packet loss that the system experienced during a pre-deployment experiment involving 22 sensor nodes. The third claim can be observed in Figure 7 where the RSSI and its standard variation is plot against time for three radio links. From all these results, it appears clearly that the RSSI value is not stable in time nor in space. These variations are due to well-known fading and shadowing effects but also to the human body which offers strong attenuation properties. For example, depending on the HCW attitude and their position in the room, RSSI variations up to $20 \mathrm{dBm}$ can be observed. Under these conditions, it is very tricky to differentiate a contact with a $\mathrm{HCW}$ in front but outside a room from a contact with a HCW inside a room but with their body between the mobile device and the fixed one.

The impact of the packet loss and the RSSI variability on the measurement reliability is obvious: it leads to false-negatives, in-room presences that are not recorded, but also false-positives, as when a $\mathrm{HCW}$ is facing the fixed sensor node from outside the room. From this pre-deployment phase, we can already draw some conclusions:

- a correct dimensioning of the neighbour protocol is required to limit the measurement system error;

- the measurements collected by such a deployment are partially erroneous and should not serve for application analysis or evaluation without being somehow corrected.

\subsection{Protocol dimensioning}

\begin{tabular}{|c|c|c|c|c|}
\hline RSSI level & 4 & 3 & 2 & 1 \\
\hline $\mathrm{dBm}$ & $\geq-60$ & $\geq-65$ & $\geq-70$ & $\geq-75$ \\
\hline
\end{tabular}

Table 1: RSSI Thresholds.

\begin{tabular}{|c|c|}
\hline$W$ & Transmission power \\
\hline $5 \mathrm{~s}$ & $0 \mathrm{dBm}$ \\
\hline
\end{tabular}

Table 2: Proximity detection parameters.

The high system loss rate combined to the usually brief presence of HCWs in rooms required the use of a short $W$ value to ensure reliability in the proximity detection system. Moreover, due to the high variations in the received signal strength, the proximity detection could not be performed using a single RSSI threshold only. Instead, we had to record more information on the received signal strength associated to each contact, i.e. each received packet. Given the sensor node memory constraints - their storage size is $1 M b$ only - and the deployment duration, recording the exact reception strength was not an option. Instead, we defined four reception levels, corresponding to four signal strength intervals, and recorded each contact together with its reception level. The protocol parameters and the reception levels that were finally used in the deployment are given in Tables 1 and 2 .

\section{Raw Data - Observations}

The resulting data consists of 16,066, 096 contacts over a period of 98 days between $56 \mathrm{HCWs}-$ 6 of the mobile sensors failed to report any data and 32 rooms. A contact $c=(t, m, f, r)$ indicates that fixed sensor $f$ received a signal from mobile 

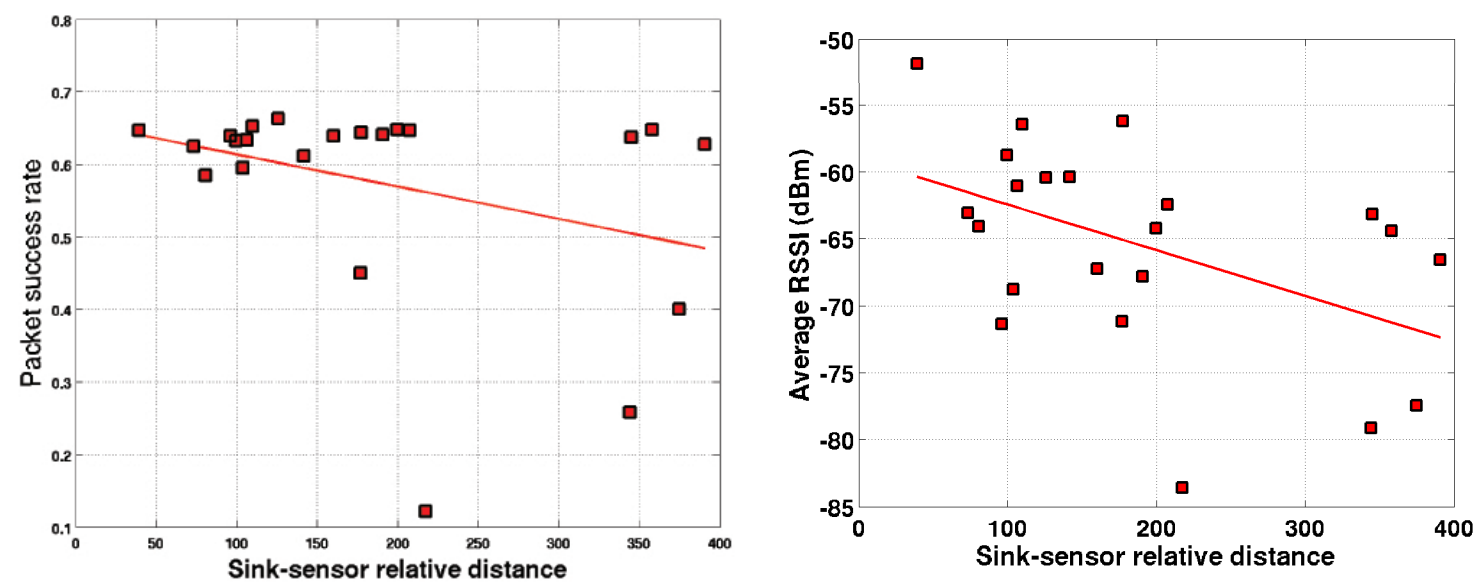

Figure 6: Average RSSI (left) and packet delivery ratio (right) versus sink-sensor relative distance.
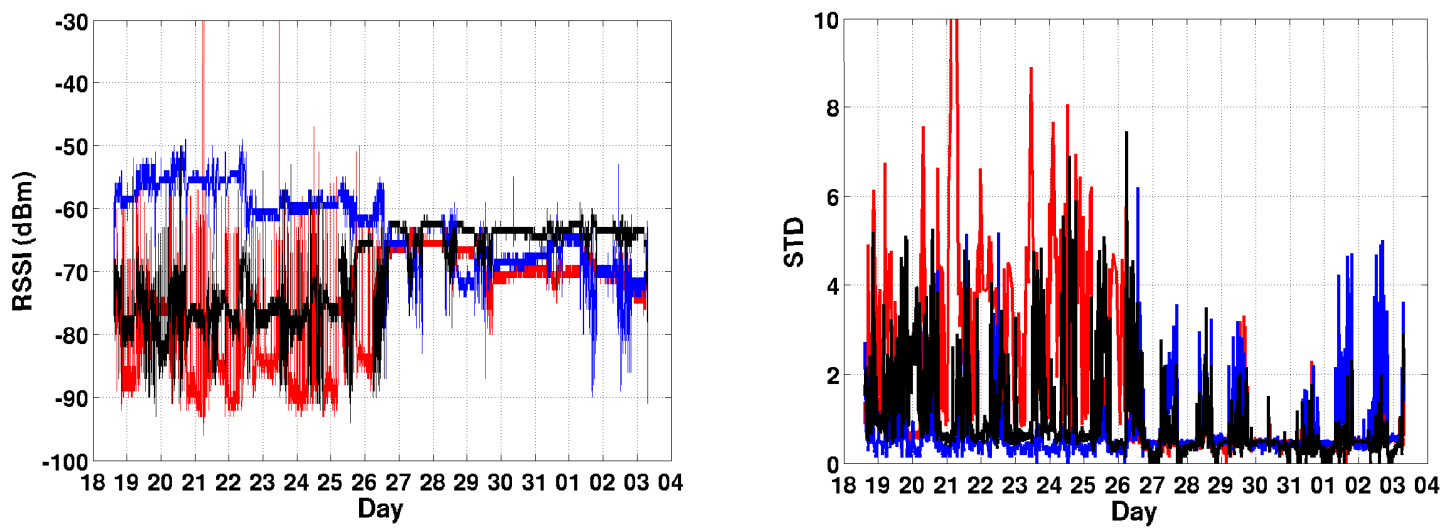

Figure 7: RSSI (left) and RSSI standard deviation (right) variations over time for three radio links.

sensor $m$ with a RSSI $r \in[1,4], 1$ being the weakest and 4 being the strongest, at time $t$.

As the measurement of contacts was made discretely, we extend the notion of contact to the one of visit $v=(t, d, m, f, r)$ where $d$ is the maximal duration of the contact. In other words, if a signal from $m$ is received by $f$ with $\mathrm{RSSI} \geq r$ every 5 seconds between $t$ and $t+d$, we consider that there is a visit of RSSI $r$ during all that period.

Looking at the data, we note that the number of contacts per fixed sensor is heterogeneous (Figure 8). The apparent absence of data for some rooms is due to the fact that their are no rooms numbered 7, 13 and 18 in the service, as shown on Figure 3. By observing the distribution of number of contacts, we were able to isolate rooms 14 and 17 from the other rooms for having an abnormally high number of contacts. It appeared that these room are next to the room where unused mobile sensors were stocked at night (Figure 3) and therefore were continuously receiving radio packets from those sensors even though no HCW was present in the room.

Out of the 30 remaining rooms (Figure 8), the number of contacts span from less than 100,000 to more than 1,000,000. Moreover, when decomposing this number by RSSI level, it is noticeable that there is no global trend : room 8 has about 100, 000 contacts of RSSI 4 (more than $10 \%$ of its contacts) whereas room 6 has less than 5,000 (less than 5\% of its contacts).

The distribution of contacts per mobile sensor (Figure 8 ) is heterogeneous as well. 4 sensors have been seen less than 1,000 times whereas all 52 oth- 

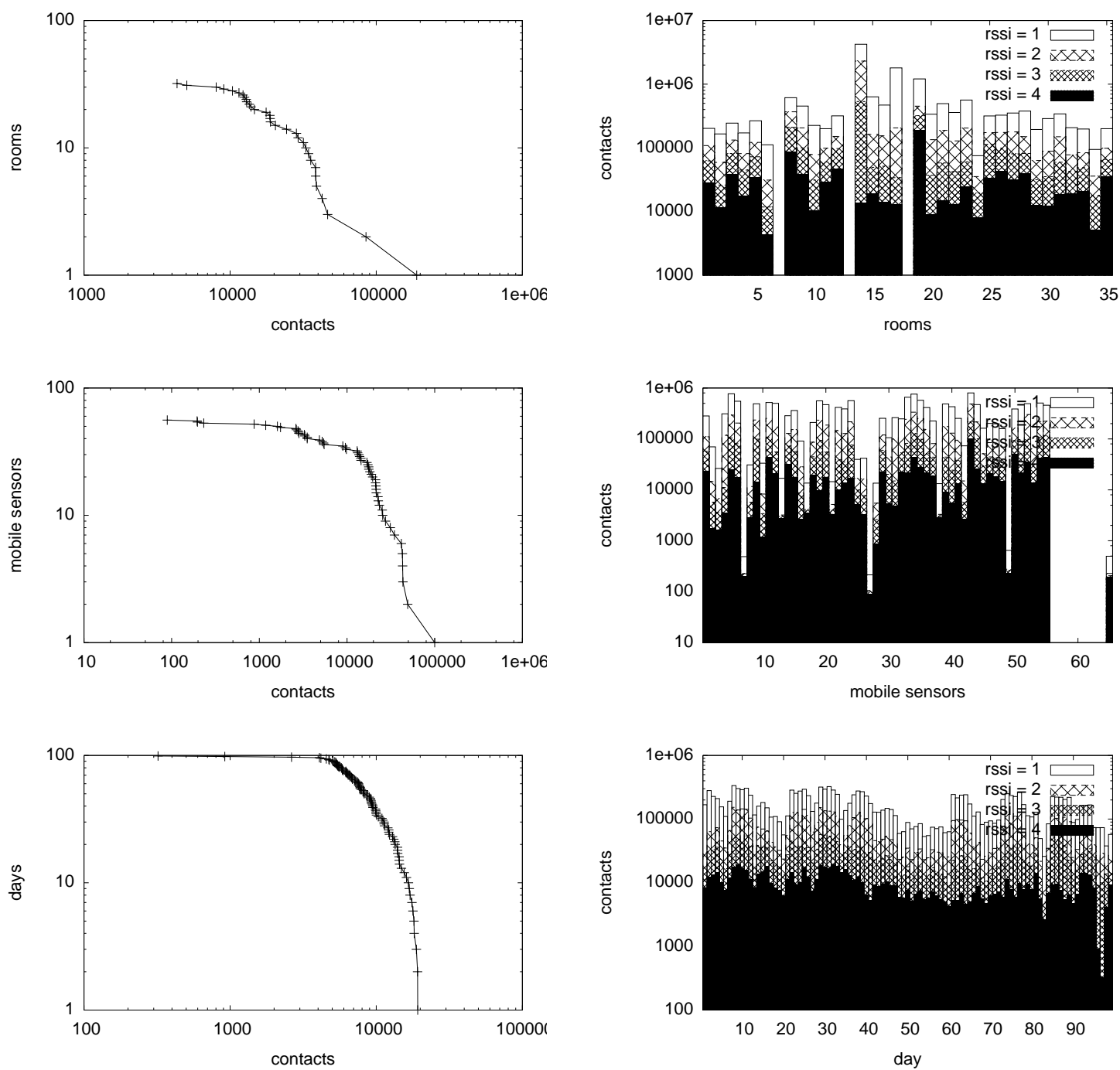

Figure 8: Number of contacts received by fixed sensors, mobile sensors, deployment day and the reversed cumulative distributions of level 4 contacts.

ers were seen at least 10,000 times (taking into account all RSSIs). These differences can be explained by the intrinsic heterogeneity of HCWs, as nurses do not have the same behaviour as doctors, interns or social assistants.

The distribution of contacts per day of deployment (Figure 8) displays a heterogeneous behavior at a daily level although weekly trends are evidenced with a lower activity towards the end of the week. Moreover, activity varies from one week to another, e.g.. weeks $7 \& 8$ (days 49 through 62) have a low activity whereas week 2 (days 7 through 14) has a higher activity. It is interesting to notice that the former two weeks were official holidays in
France, probably with less HCWs present in the service.

In the rest of the article, we focus on a subset of the data consisting of the interactions between all HCWs and 63 patients monitored for being potentially infected by tuberculosis.

\section{Error model}

\subsection{False negatives}

Packet loss. We call intercontact the duration between two consecutive contacts between a same couple (fixed, mobile) of sensors. This duration repre- 

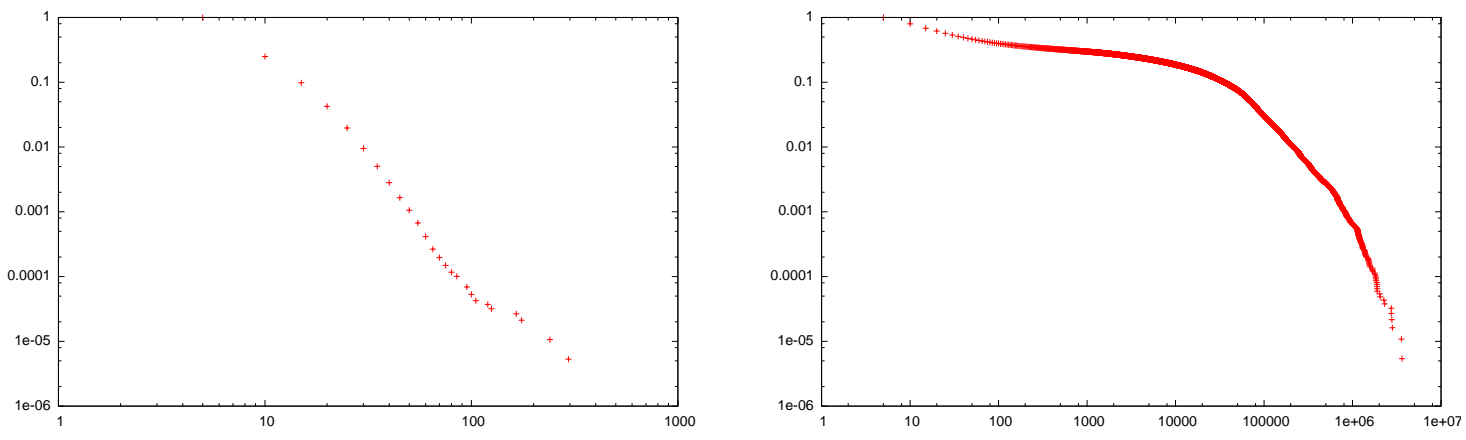

Figure 9: Distributions of visits durations (left) and intercontacts (right).

sents the time during which a $\mathrm{HCW}$ is absent from a room before re-entering.

By observing the distributions of both visits and intercontacts durations (Figure 9), we notice that $90 \%$ of all visits last at most 10 s and that moreover $30 \%$ of intercontacts last less than 15 s. Those two observations are blatantly erroneous and can be explained by the volatility of the radio medium. Loss of packets induces false negatives in the measurement.

Assuming a uniform and stationary packet loss, we propose a method to evaluate the probability $p$ of receiving a packet based on the intercontacts distribution and further confirmed by the audits.

Probability of measuring a contact. It is legitimate to suppose that there exists a minimal intercontact duration which corresponds to the fact that a HCW does not exit a room only to re-enter a few seconds later. We suppose that this duration is at least equal to $20 \mathrm{~s}$.

As a consequence, we can consider that intercontacts of less than 20s are due to a packet loss, and thus the probability of measuring an intercontact of $5 k$ seconds is exactly the probability of losing $k$ consecutive packets. Hence, assuming a uniform and stationary loss, $(1-p)^{k}$. Using the measured data, we estimate $p \sim 0.13$.

Validation using the audits. By comparing the audits and the data obtained during the same period, we can evaluate $p$ as being the fraction of measured presence per effective presence. We estimate by this method that $p \sim 0.145$ which is coherent with the value obtained by the observation of short intercontacts.

\subsection{False positives}

The existence of a contact does not guarantee an actual presence in the room. Due to the nature of the radio medium, the signal from a HCW walking in front of an opened door or in an adjacent room might be received with a higher strength than that of a $\mathrm{HCW}$ in the room but turning its back to the fixed sensor. Although the probability of having a false positive might be small, it is important to keep in mind that because of the great number of contacts, a lot of false positives might arise. We assume that the probability of measuring a false positive is uniform and stationary, hence the probability of having $k$ consecutive contacts decreases exponentially with $k$.

\section{Reconstruction method}

Due to these observations, reconstructing the original signal is a necessity, as the measured data does not represent actual social interactions. We thus propose the following reconstruction protocal, illustrated in Figure 10:

- reveal false negatives:

- determine the minimal intercontact duration $d_{i}$;

- aggregate successive contacts separated by at most $d_{i}$ seconds;

- delete false positives:

- determine the minimal visit duration $d_{v}$

- remove all visits of less than $d_{v}$ seconds 

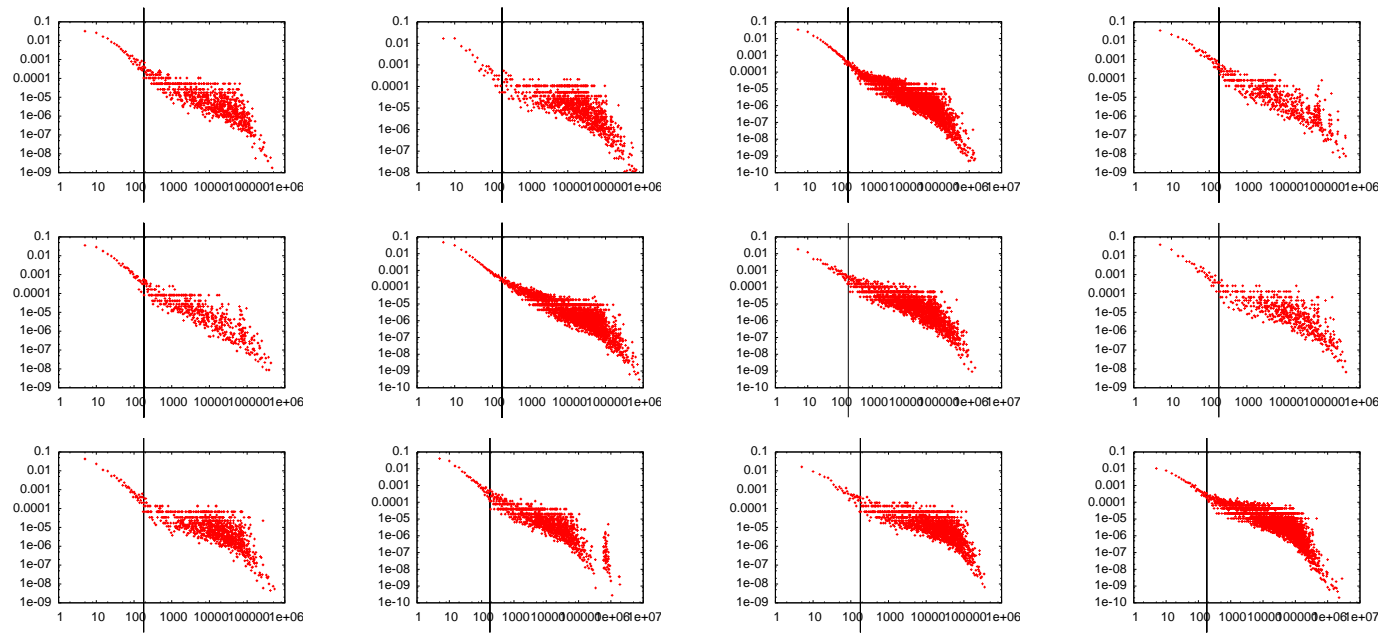

Figure 11: Right derivative of the reversed cumulative distribution of intercontacts durations in different rooms. The vertical line indicates $180 \mathrm{~s}$.

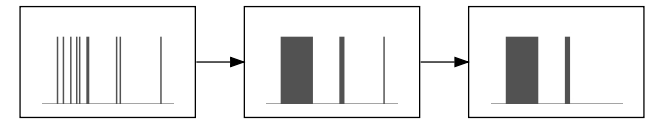

Figure 10: Illustration of the reconstruction protocol.

\subsection{Minimal intercontact duration}

As explained before, short intercontacts are solely a consequence of the packet loss. By observing the distributions of intercontacts durations for each room, we note on Figure 11 a change of behavior (inflection of the derivative of the reversed cumulative distribution function) around 180s. The change represents the fact that longer intercontacts are not only due to packet loss but also to the HCW behavior and thus to actual intercontacts. Hence, we consider $d_{i}=180$.

\subsection{Minimal visit duration}

We first aggregate all contacts separated by at most $d_{i}$ seconds and plot, for each duration $d$ and each room $r$ the fraction of the total presence duration in room $r$ due to visits of duration $d$ as a function of the proportion of visits of duration $d$ in this room. This represents the relationship between contributions in terms of duration and number of visits of each duration. On Figure 12 we observe that the visits of 5 seconds, highlighted on the figure and contained in the right bow area, display

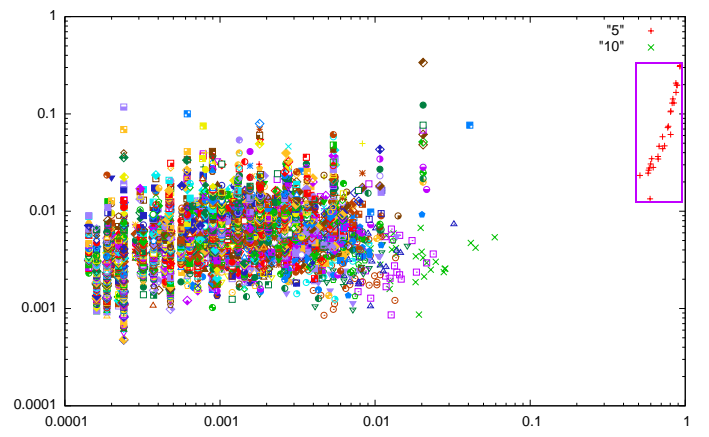

Figure 12: Contributions of visits, per room and duration, to the total of visits and durations.

a totally different contribution than that of other durations, and we conclude that the minimal visit duration is at least 10 seconds. Given that there is no clear separation between the contributions of visits of more than $10 \mathrm{~s}$, we assume $d_{v}=10$.

\section{Results}

\subsection{Intercontacts}

After reconstruction, we obtain the distribution of intercontacts given in Figure 13. Its apparent irregularity, which was not present beforehand, is explained by the work schedule of HCW and in particular by the fact that the maximum work (respectively rest) period per day is around $8 \mathrm{~h}$ (resp. 16h). Thus, for example, there are very few intercontacts which duration is more than $8 \mathrm{~h}$ and less than $16 \mathrm{~h}$. 

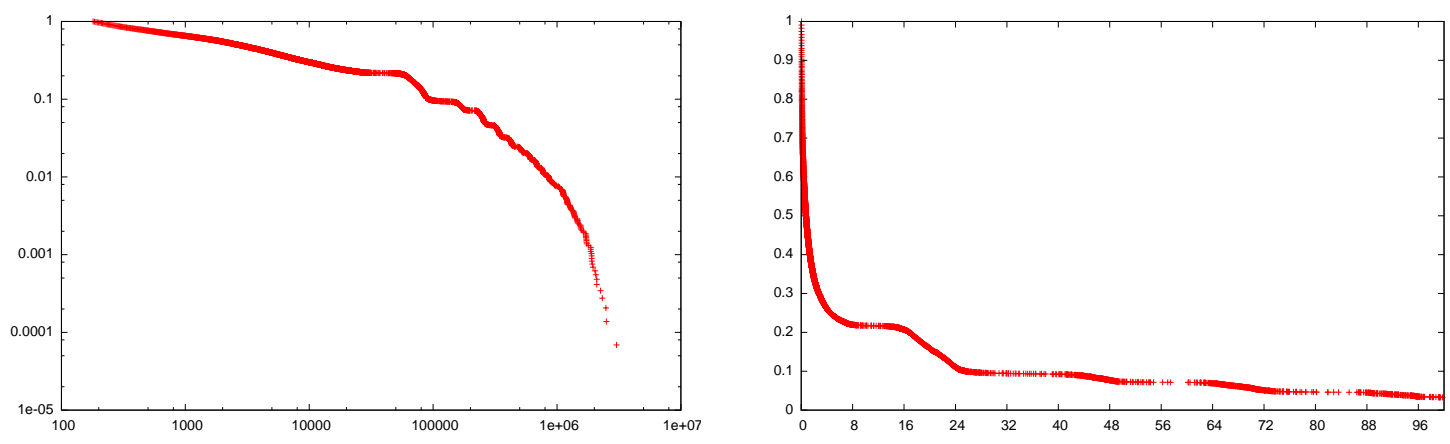

Figure 13: Distribution of intercontacts durations after reconstruction (left) and zoom on the first 100 hours (right).

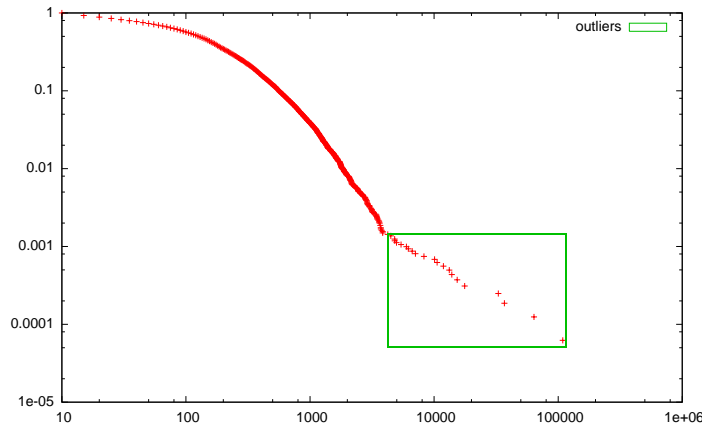

Figure 14: Distribution of visits durations after reconstruction.

This observation reinforces the idea that the reconstruction method is correct, as it brings out retrospectively a real behavior which was not explicit in the original distribution and which is not due to a reconstruction bias.

\subsection{Visits}

The distribution of visits durations (Figure 14) clearly highlights the presence of outliers, i.e. visits which durations are abnormally high. These 23 outliers are divided as follow: 19 visits of doctors in room 19 (next to a room used by doctors for computer work), 3 visits of a nurse in room 14 (next to the room where sensors were stocked when not used) and one visit of a nurse in room 8 (adjacent to the room where HCW stayed while not in the patient rooms). Given that those outliers' existence can be explained by the service's topography or by the negligence of a HCW (forgetting a blouse in a room, for example), we consider that they are not a reconstruction error and propose their deletion from the dataset.

\subsection{Some statistics}

In order to exhibit the differences induced by the reconstruction we present in Table 3 a comparison of several statistics on the number and durations of visits to selected patients before and after reconstructing the presence signal. It makes no doubt that any further exploitation of the HCW visits data will be altered depending on wether they would have been based on the raw measures or the reconstructed interactions.

\section{Conclusion}

The data collected within the framework of the TubExpo project is huge and unique in nature, consisting in the record of the HCWs presence in all patient rooms of the SMIT unit, 24 hours a day, 7 days a week, during a three months period and on a $5 \mathrm{~s}$ basis. Using this data set, we have highlighted the bias introduced by the measurement system, mainly caused by the radio medium versatility, and we have argued that this bias can lead to major miss-interpretation of the data and/or wrong model behaviors. In order to correctly exploit the data and recover from measurement errors, we have devised a method that can be used to reconstruct the original interactions information and which uncovers phenomena which were not visible on the raw data.

Now that the issue of measurement reliability has been clearly identified, many points remain as future extensions of this work. Alternate reconstruction algorithms can be investigated and in particular, an individual analysis leading to a specialization of the reconstruction parameters to the different sensor nodes appears as a promising strategy. Further developments will also be oriented 


\begin{tabular}{|c|c|c|c|c|c|c|c|c|c|c|c|c|}
\hline Patient & \multicolumn{2}{|c|}{ Nb of visits } & \multicolumn{2}{l|}{ shortest (s) } & \multicolumn{2}{l|}{ longest (s) } & \multicolumn{2}{|c|}{ average duration (s) } & \multicolumn{2}{|c|}{ std dev } & median (s) \\
\hline 25 & 1089 & 75 & 5 & 10 & 30 & 640 & 5.50 & 140.13 & 2.02 & 151.38 & 5 & 100 \\
26 & 3860 & 368 & 5 & 10 & 40 & 3850 & 6.74 & 283.65 & 3.79 & 421.94 & 5 & 135 \\
27 & 1849 & 169 & 5 & 10 & 60 & 2155 & 7.59 & 233.58 & 5.67 & 329.85 & 5 & 115 \\
28 & 1148 & 120 & 5 & 10 & 50 & 2415 & 6.96 & 309.25 & 4.52 & 411.68 & 5 & 170 \\
29 & 7639 & 684 & 5 & 10 & 55 & 3160 & 7.26 & 277.76 & 4.73 & 327.60 & 5 & 175 \\
30 & 11157 & 1151 & 5 & 10 & 60 & 3600 & 7.47 & 252.72 & 4.92 & 370.92 & 5 & 125 \\
31 & 1911 & 143 & 5 & 10 & 60 & 2175 & 6.94 & 162.55 & 5.44 & 251.00 & 5 & 95 \\
32 & 2105 & 166 & 5 & 10 & 30 & 985 & 5.54 & 126.96 & 2.16 & 155.41 & 5 & 75 \\
33 & 5392 & 441 & 5 & 10 & 35 & 1255 & 5.79 & 156.35 & 2.60 & 184.00 & 5 & 100 \\
34 & 3231 & 365 & 5 & 10 & 80 & 1600 & 7.79 & 201.03 & 6.69 & 225.85 & 5 & 135 \\
\hline
\end{tabular}

Table 3: Various statistics before (left) and after (right) reconstruction for a subset of the patients

towards analyzing the TubExpo data set with respect to additional health care information related to the patients that was collected during the experimentation. We have to address more sophisticated models both from the clinical/health care perspective, e.g. epidemic models, health care strategies or public health policies, and from the network analysis perspective in order to answer the fundamental questions that have originally motivated this work.

\section{Acknowledgments}

This work was supported by a public grant from the French National Agency for Food, Environmental and Occupational Health Safety (ANSES/AFSSET, EST 2007-50)

\section{References}

[1] H. Alani, M. Szomsor, C. Cattuto, W. Van den Broeck, G. Correndo, and A. Barrat. Live social semantics. In 8th International Semantic Web Conference (ISWC2009), Washington DC, USA, October 2009.

[2] R. Albert, H. Jeong, and AL. Barabási. The diameter of the World Wide Web. Nature, 401:130-131, 1999

[3] E. Ben Hamida, G. Chelius, A. Busson, and E. Fleury. Neighbor discover $\mathrm{y}$ in multi-hop wireless networks: evaluation and dimensioning with interference considerations. Discrete Mathematics and Theoretical Computer Science, 10(2):87-14, 2008.

[4] A.Z. Broder, S.R. Kumar, F. Maghoul, P. Raghavan, S. Rajagopalan, R. Stata, A. Tomkins, and J. L. Wiener. Graph structure in the web. Computer Networks, 33, 2000.

[5] A. Carbonne, C. Poirier, G. Antoniotti, C. Burnat, C. Delacourt, C. Orzechowski, P. Astagneau, and E. Bouvet. Investigation of patient contacts of health care workers with infectious tuberculosis: 6 cases in the paris area. The International Journal of Tuberculosis and Lung Disease, 9(8):848-852, 2005.

[6] C. Cattuto, A. Barrat, and W. Van den Broeck. http://www.sociopatterns.org/.
[7] CSHPF. Enquête autour d'un cas de tuberculose: recommandations pratiques. Conseil supérieur d'hygiène publique de France, 2006.

[8] M. Faloutsos, P. Faloutsos, and C. Faloutsos. On powerlaw relationships of the internet topology. In $A C M S I G$ COMM, 1999.

[9] A. Fraboulet, G. Chelius, and E. Fleury. Worldsens: development and prototyping tools for application specific wireless sensors networks. In International Symposium on Information Processing in Sensor Networks (IPSN), Boston, USA, April 2007. ACM.

[10] P. Hui, A. Chaintreau, J. Scott, R. Glass, J. Crowcroft, and C. Diot. Pocket switched networks and human mobility in conference environments. In SIGCOMM, Philadelphia, USA, August 2005. ACM.

[11] J. M. Kleinberg, R. Kumar, P. Raghavan, S. Rajagopalan, and A. S. Tomkins. The web as a graph: Measurements, models, and methods. In COCOON, 1999.

[12] R. Kumar and M. Latapy. Theoretical computer science special issue on complex networks - foreword. Theoretical Computer Science, 355(1):1-5, April 2006.

[13] Mark Newman, Albert-Laszlo Barabási, and Duncan J. Watts. The Structure and Dynamics of Networks. Princeton University Press, Princeton, USA, 2006.

[14] A. Seidler, A. Nienhaus, and R. Diel. Review of epidemiological studies on the occupational risk of tuberculosis in low-incidence areas. Respiration, 72:431-446, 2005.

[15] D. Watts and S. Strogatz. Collective dynamics of 'smallworld' networks. Nature, 393:440-442, June 1998. 